\title{
COVID-19 Induced Cardiovascular Complications and Recent Therapeutic Advances
}

\author{
Selia Chowdhury, Mehedi Hasan Bappy, Samia Chowdhury, Md. Shahraj Chowdhury, \\ and Nurjahan Shipa Chowdhury
}

\section{ABSTRACT}

A global pandemic instigated by SARS-CoV-2 virus has been going strongly for almost two years. There have been almost 246 million cases with almost 5 million fatalities all over the world so far. COVID has affected almost every aspect of people's lives halting the world in immeasurable ways. Although COVID-19 is mostly affecting the respiratory system, it is also responsible for a significant amount of cardiovascular system involvement. On one hand, it deteriorates any pre-existing cardiovascular condition, on another hand, it triggers other inflammation-facilitated acute events. They comprise of inflammatory heart disease, severe pericardial effusion, ventricular arrhythmias, endothelial dysfunction, pulmonary hypertension, heart failure, and many more. In this article, we summarize the features, management, and treatment of several important COVID-19 induced cardiovascular complications. Recent advances in the treatment of these diseases along with the justification and concerns of specific therapeutic measures are also discussed.

Keywords: Cardiovascular, management, therapeutics, COVID-19.

Submitted: October 10, 2021

Published: November 13, 2021

ISSN: 2593-8339

DOI: $10.24018 /$ ejmed.2021.3.6.1099

S. Chowdhury*

Dhaka Medical College, Dhaka, Bangladesh.

(e-mail: chowdhury.selia@gmail.com)

M. H. Bappy

University of Iowa, Iowa City, IA, USA.

S. Chowdhury

Sylhet MAG Osmani Medical College,

Sylhet, Bangladesh.

M. S. Chowdhury

DSHE, Education Ministry, Sylhet, Bangladesh. N. S. Chowdhury

Dinajpur Medical College, Dinajpur, Bangladesh.

*Corresponding Author

\section{INTRODUCTION}

COVID-19 has been a nightmare to billions of people all over the world impacting their lives with unprecedented losses be it economic, physical, or mental. As of October 26, 2021, there happened about 246 million cases and death toll reached approximately 5 million; United States, India, Brazil, United Kingdom, and Russia are leading the list for most COVID cases [1]. Although there have been vaccines which are very effective to protect against coronavirus, the number of cases and deaths are still rising. One critical consequence of this infection is its multisystem involvement in human body. The impact of COVID-19 is not only confined within the respiratory system but other body systems and organs such as the nervous system, eye, gastrointestinal tract, renal and urinary structures, hemopoietic, and cardiovascular system are also severely affected [2].

The SARS-CoV-2 virus has several life-threatening effects on the cardiovascular system, it worsens any pre-existing cardiovascular condition as well as triggers other inflammation-facilitated acute events. A variety of cardiovascular complications have been reported so far due to SARS-CoV-2 and they have triggered a large range of complications resulting in high morbidity and mortality. Recent articles demonstrate that COVID-19 bring about serious cardiovascular complications, namely ventricular arrhythmias, thrombotic events, myocardial infarction (MI), myocarditis, heart failure (HF), cardiogenic shock, and sudden cardiac death (SCD) [3]-[5]. Several treatments, therapeutic options, and management guidelines are available for these conditions. A comprehensive cardiovascular workup is essential in COVID-19 patients with cardiorespiratory symptoms to properly diagnose and manage them. This review article outlines and discusses several COVID-19 induced cardiovascular complications, their pathophysiological features, and clinically tested possible treatment options that have promise of being advantageous in COVID-19 patients with cardiovascular complications. The complications discussed here are Myocarditis, pericardial effusion, Pulmonary hypertension, Endothelial dysfunction, Cardiac arrhythmia, and Heart failure.

\section{Myocarditis}

Myocardial injury is directly associated with higher mortality and is a poor prognostic factor in patients with COVID-19. According to numerous published case series, a significant amount of acutely ill COVID-19 patients who requires intensive care is characterized by elevated troponin levels in respect to myocardial injury. Several causes of myocardial injury related to COVID-19 have been described such as straight myocardial injury by SARS-CoV-2, stress cardiomyopathy, pro-thrombotic and microvascular disease, mismatch of oxygen demand to supply, and a systemic hyperinflammatory response with myocardial participation [6], [7]. These diverse sources of myocardial injury are repeatedly 
referred to as myocarditis, even though myocarditis, in a precise sense, describes direct viral injury and accompanying inflammation [8]. Myocarditis due to COVID-19 has been reported but it is less frequent, and coronavirus has not been formerly considered cardiotropic [8]. However, concern for COVID-19 associated myocarditis has heightened as the pandemic death tally related to respiratory and cardiovascular complications is increasing day by day [9], [10].

Guo et al. [8] discussed the available medicinal options and management of patients with myocarditis who are infected with SARS-CoV-2. It is unclear that if the clinical, imaging, histochemical, and prognostic indications from other types of viral myocarditis can also be applied in COVID-19 associated myocarditis or not. Furthermore, therapeutic alternatives which are advantageous in COVID-19 connected myocarditis are uncertain. As soon as myocarditis has been manifested, immunosuppression may be uncovered, specifically in patients hospitalized with increased systemic inflammation and hypoxemia, although statistics supporting this methodology are deficient [8]. Currently, management of myocarditis in COVID-19 patients follows standard heart failure guidelines. Specific therapies in SARS-CoV-2 myocarditis are not sufficiently supported by evidence. In the situation of simultaneous pneumonia, dexamethasone can be prescribed to patients with hypoxemia and IL-6 antagonists are acceptable for critically ill patients who have a marked systemic inflammatory response. Corticosteroids could be considered in isolated SARS-CoV-2 myocarditis patients who are hypoxemic or hospitalized. However, for patients with a less severe conditions, corticosteroids are preferred to be avoided. Concerning targeted immunomodulatory therapy with IL-1 or IL-6 antagonists, additional evidence-based data are considered necessary before use could be recommended specifically for SARS-CoV2-myocarditis [8].

\section{PERICARDIAL EFFUSION}

On top of pulmonary indications, the virus can have detrimental consequences on other body parts, notably heart. As a perilous and life-threatening complication in patients with COVID-19 large pericardial effusion has been observed [11]. The principal mechanisms responsible for this phenomenon are cited as direct cardiomyocyte and pericardial invasion, inflammation, oxidative stress due to acute respiratory distress syndrome, and cytokine storms [11]. Pericardial effusion can occur as a prolonged difficulty along with myocarditis or pericarditis, with acute respiratory distress syndrome or both isolated.

Three types of pericardial effusion have been observed in patients infected with COVID-19, they are serosanguinous, serous, and bloody. Hemorrhagic pericardial effusion needs intensive treatment as it may progress to develop constrictive pericarditis as a complication or even recur [12]. Evaluation of albumin levels and lactate dehydrogenase suggested that most of the pericardial effusion cases connected to COVID19 are exudative [13]-[15] however, a transudate pattern has also been noticed [16].

According to size in patients of COVID-19, Pericardial effusion might be classified as mild, moderate, and large. Medications can be used in the case of mild and moderate pericardial effusion, particularly in patients with pericarditis pathology. There is no specific guideline for the management of COVID-19 patients with pericardial effusion. Nevertheless, only colchicine or colchicine in conjunction with other anti-inflammatory medications, has been found to be helpful [13]. The statements on the subject of using NSAIDs in COVID-19 patients is conflicting between World Health Organization (WHO) and European Medicines Agency (EMA). While WHO says using NSAIDs facilitates virus penetration into cells by enhancing ACE2 receptor expression [17], EMA finds no scientific evidence supporting this [14]. Ibuprofen has been the most used NSAID, it did not have any acute side effect. For pericarditis and pericardial effusion with COVID-19, Colchicine has been nominated as the first drug which can reduce inflammation. Additionally, in patients who are intolerant/irresponsive to NSAIDs, oral steroids can be an alternative. Especially for critically ill patients, intravenous steroids are recommended [18]. Combinations of steroids and colchicine may also help to prevent a recurrence. However, patients who are hemodynamically unstable with moderate or large effusion call for emergency drainage [16]. The drainage can be via surgery or percutaneous pericardiocentesis as these techniques result in speedy relief of effusion. The more conventional method in most inpatient settings is percutaneous pericardiocentesis, it can be performed with fluoroscopic or echocardiographic guidance [19]. Besides, surgical drainage is the desired method in patients with coagulopathy and a biopsy is favored for diagnostic reasons [20].

\section{PUlmonary HyPertension}

An uncommon demonstration of acute respiratory distress syndrome (ARDS) is featured in COVID-19 patients by recent clinical reports. Severe hypoxemia, cytokine release storm, imbalance of the renin-angiotensin system, and increase in thrombogenic processes singularize it. These processes both aggravate lung injury and foster vasoconstriction and pulmonary vascular remodeling leading to pulmonary hypertension (PH) [21].

It is suggested that a multidisciplinary team consisting of an intensivist, pulmonologist, cardiologist, and infectious disease specialist should be consulted to manage COVID-19 patients with $\mathrm{PH}$ [22]. In case of clinical evidence of heart failure or RV dysfunction by cardiac magnetic resonance imaging (CMR), patients should be managed according to guideline-directed medical therapy [23]. Numerous clinical trials utilizing drugs with capacities to impact pulmonary vasoconstriction are currently in progress. Al-Kuraishy et al. concluded in their study that Phosphodiesterase Enzyme Type 5 Inhibitors (PDE5Is) hold a significant part in the management of COVID-19 patients with ARDS through mitigations of inflammatory changes and associated coagulopathy [24]. Hence, PDE5Is should be advised in the basic therapeutic procedure to avert pulmonary as well as cardiovascular complications. Feng et al. [25] reported the possibility of treatment with inhaled nitric oxide (iNO) in the COVID-19 patients with pulmonary hypertension. In wellventilated lung tissue, inhaled NO specifically expands the 
pulmonary arteries and has no impact on breath perfusion contrary to orthodox vasodilators like sildenafil or nifedipine. Thus, they enhance blood oxygenation and decrease the intrapulmonary shunting of blood from right to left [26]. They indicated that use of inhaled NO was helpful in decreasing and stabilizing the pulmonary arterial systolic pressure in case of seriously ill COVID-19 patients who have PH. It was also beneficial in reducing the danger of heart failure in COVID-19-related ARDS patients. Therefore, inhaled NO is reasonable to consider as a prospective therapeutic choice for these patients.

\section{ENDOTHELIAL DYSFUNCTION}

COVID-19 engenders the risk of several non-pulmonary issues like thromboembolic events and acute myocardial injury, acute Endothelial Dysfunction (ED) and injury may explain these phenomena. ED is a familiar property of the comorbidity factors which enhance the danger of severe COVID-19, namely obesity, hypertension, coronary artery disease, heart failure, and diabetes mellitus. Relevant research imply that SARS-CoV-2 may infect vascular endothelial cells; prevalent endothelial damage and inflammation is found and reported in severe cases of COVID-19. Vexation of ED in COVID-19 can damage organ perfusion to initiate a procoagulatory state. It is due to the vital function of endothelial cells in sustaining \& controlling vascular homeostasis and blood coagulation causing macrovascular and microvascular thrombotic events [27].

Reference [27] described therapeutics and management guidelines regarding endothelial dysfunction. They suspect RAS inhibitors to possibly be effective in prevention and management of COVID-19 related systemic complications through the improvement of endothelial dysfunction. Current evidence suggests that in absence of serious contraindications, it may be recommended to continue RAS inhibitors and statins in COVID-19 patients. For prevention, current guidelines suggest for patients with cardiovascular symptoms ought to be recommended RAS inhibitors and statins as well as to follow best possible healthy lifestyle [27].

\section{CARDIAC ARRHYTHMIA}

COVID-19 is known to be related to an enhanced incidence of arrhythmia in hospitalized patients. In $16.7 \%$ of hospitalized COVID-19 patients, cardiac arrhythmia was reported, and it influenced $44 \%$ of those shifted to the intensive care unit [28]. Guo et al. [8] concluded in their study that elevated levels of troponin-T (TnT) was exhibited in patients with underlying CVD leading to more frequent development of complications including malignant arrhythmias and ventricular tachycardia/fibrillation. However, patients without prior history of CVD also can express elevated levels of $\mathrm{TnT}$, and develop malignant arrhythmias, but it is less frequent (TnT levels were found to be raised in $13.2 \%$ of patients without any underlying CVD compared to $54.5 \%$ of patients with underlying CVD; malignant arrhythmias occurred in $5.2 \%$ of patients without CVD compared to $11.5 \%$ in patients with CVD) [29]. Cardiac arrhythmias were detected two-fold more frequently at high
Troponin levels [29]. Myocardial tissue damage could be direct or indirect through multiple mechanisms including the receipt of proarrhythmic drugs (chloroquine, hydroxychloroquine, or azithromycin), cytokine storm, electrolytes imbalances in hospitalized patients, right ventricular strain secondary to pulmonary hypertension or pulmonary embolism, and endogenous catecholamine adrenergic status may source the development of arrhythmia [30].

Hydroxychloroquine (HCQ) blocks Kv11.1 (HERG) and can result in development of drug-induced Long QT syndrome [31]. The clinical arrhythmic toxicity is mostly limited to chronic use (since it has a prolonged half-life of 40 days), use of multiple QT-prolonging medications (azithromycin) at the same time, metabolic imbalance, renal insufficiency, or in case of acute overdose [32], [33]. Since the suggested hydroxychloroquine therapy for COVID-19 is quite short (5-10 days), the risk of arrhythmia is expected to be less. However, specific precautions should be considered for patients with congenital long QT syndrome, severe renal failure, and electrolyte derangements (hypokalemia and hypomagnesemia). It is suggested that all QTc-prolonging medications that are not essential should be identified and discontinued. Electrolytes should be monitored and maintained within normal reference range (potassium $>4.0$ $\mathrm{mmol} / \mathrm{L}$, magnesium $>1 \mathrm{mg} / \mathrm{dL}$, and calcium 2.15-2.5 $\mathrm{mmol} / \mathrm{L}$ ) [34]. Baseline renal and hepatic function should also be obtained and monitored as in patients with acute kidney injury, chronic kidney disease, and hepatic insufficiency an inadequate adjustment of dose of renal/hepatic eliminated drugs may lead to elevated plasma levels causing an enhanced risk of drug-induced Torsade de Pointes and/or Sudden Cardiac Death. A baseline ECG should be done and the QTc should be calculated by the traditional method before starting treatment [34].

\section{HEART FAILURE}

Because of SARS-CoV-2 infection, heart failure (HF) has become an increased threat. Especially in older patients who have inherent comorbidities like ischemic heart disease, hypertension, and diabetes mellitus [35]. Cardiac injury biomarkers have been found to be higher in severely ill COVID-19 patients, which suggests the progress of direct acute cardiac injury or indirect injuries owing to pulmonary complications, for instance, ALI and ARDS [36]. It has been conjectured that in the early stage of infection, acute cardiac injury results in a typical HF with sustained ejection fraction, on the other hand, in the subsequent stage of infection, HF together with systolic dysfunction is developed owing to evolution of cytokine storm [37]. Cardiac ACE2 expression is associated as a target for COVID-19 induced intense cardiac injury. Like other febrile illnesses, in SARS-CoV-2 infection, multisystem inflammatory syndrome, high blood viscosity, endothelial dysfunction induced coagulation disorders, and amplified pro-inflammatory response could contribute to the increased risk of HF development [35]. Because of the progression of pulmonary hypertension, heart hemodynamic stability may also get affected by hypoxic respiratory failure, like in pulmonary edema, acute 
respiratory distress syndrome (ARDS), and severe acute lung injury (ALI). Furthermore, HF induce by COVID-19 may well be within the growth of cytokine storm, characterized by elevated rise of pro-inflammatory cytokines which weaken cardiomyocyte function and cardiac contractility. There is a conclusive relationship between pro-inflammatory cytokine and myocarditis-induced acute cardiac injury biomarker levels in cytokine storm-mediated cardiac dysfunction [35]. Therefore, it can be said that HF induced by COVID-19 is additionally complicated and linked by a molecular setting in discharging pro-inflammatory cytokines to the neurometabolic disorders which collectedly impact cardiomyocyte tasks and progression of $\mathrm{HF}$.

Digoxin is medication that is routinely used in the management of HF and atrial fibrillation that works through the inhibition of $\mathrm{Na}^{2+} / \mathrm{K}^{+}$ATPase in the heart and subsequently increases cardiomyocyte $\mathrm{Ca}^{2+}$, which in turn improves cardiac contractility [35]. In COVID-19 the prospective function of digoxin is primarily because of the anti-inflammatory and antiviral effects: it exerts antiinflammatory and anti-viral actions counter to SARS and MERS CoV by the inhibition of mRNA transcription, protein translation, and viral particle release [38]. The principal process of the anti-inflammatory influence of digoxin is the suppression of pro-inflammatory cytokines using the NF- $\mathrm{BB}$ inhibition-dependent pathway [39]. The progression of cytokine storms in SARS-CoV-2 infection might thus be inhibited by the anti-inflammatory effects of digoxin. Moreover, digoxin controls the unregulated production of immunoglobulins and activation of $\mathrm{T}$ cells through the induction of regulatory $\mathrm{T}$ cells [40]. Thus, digoxin may prevent cardiomyocyte injury caused by autoimmune myocarditis along with the development of HF.

Carvedilol is a non-selective $\beta$ and $\alpha-1$ adrenoceptor blocker which downregulates cardiac ACE2 and prevents acute cardiac injury induced by SARS-CoV-2 [41]. In addition, carvedilol inhibits the release and activity of IL-6 [42], thus prevents tissue injury and cytokine storms development. Moreover, mineralocorticoid receptor blockers, such as eplerenone and spironolactone that are used in the management of HF, also have effects against SARSCoV-2 and can be helpful in prevention of acute complications of COVID-19, including HF [43].

\section{EFFECT OF VACCINATION ON CARDIOVASCULAR SYSTEM}

Vaccine is a pivotal tool to mitigate the current global COVID-19 pandemic. COVID-19 vaccines were found to be secure and functional in putting a stop to symptomatic, laboratory-confirmed, COVID-19 cases in multiple large, randomized controlled trials. Many unfavorable effects related to vaccines have also been reported, however. Myocarditis is one of the potential complications after administration of mRNA COVID-19 vaccines (i.e., PfizerBioNTech and Moderna) [44]. A large number of cases of possible pericarditis and myocarditis have been reported to the Vaccine Adverse Event Reporting System (VAERS) in the USA. Young and adolescent males dominate these adverse cases, and it is evidently more common after the second dose of vaccination [45]. The cases are very scarce (about 12.6 cases per million second doses administered) compared to the hundreds of millions of doses administrated so far but it deserves special attention due to the potential implications. According to the literature, the cases generally are accompanied by pyrexia, pain in the chest, and shortness of breath with changes on the ECG, and CMR findings consistent with myocarditis. However, the clinical features do not sustain for a long time but resolve rapidly. The management of the patients relates to use of mostly nonsteroidal anti-inflammatory drugs (NSAIDs), but corticosteroids and intravenous immune globulin (IVIG) were also required in some cases [46]. It is recommended to avoid competitive sports for at least 3 months until cleared by a cardiologist so that any unexpected cardiac events can be avoided while the cardiac muscle recovers [47]. Bozkurt et al. [44] discussed possible management strategies of myocarditis. Clinicians should be conscious of the risk of myocarditis and pericarditis, especially in the younger population suffering from chest pain within a week after vaccination. Initial assessment should be done by obtaining ECG and cardiac troponin levels and inflammatory markers such as CRP and ESR can also be useful. In any suspected cases consultation with cardiologist and evaluation with echocardiography and cardiac MRI should be considered. Additionally, an assessment for acute COVID-19 infection (via PCR of respiratory sample) and past disease (via detection of antibodies against SARS-CoV-2 nucleocapsid and spike protein) would be helpful. Assessment and treatment may be different based on the patient's age, clinical manifestations, other etiologies and comorbidities, hemodynamic and rhythm stability, and clinical course. They mentioned that patients with chest pain, evidence of myocardial injury, changes in ECG, abnormality in cardiac imaging, conduction defect, hemodynamic instability after administration of COVID-19 vaccine are expected to involve hospitalization and follow-up.

Vaccine-induced thrombotic thrombocytopenia (VITT) is another serious complication of COVID-19 vaccination. Usually, VITT is characterized by thrombus formation in atypical sites and concomitant thrombocytopenia [48]. The atypical sites may include thrombosis in cerebral venous sinus or thrombosis in the portal, hepatic, or splanchnic veins. The reported cases were studied after administration of adenovirus-based vaccines (i.e., AstraZeneca or Janssen) in patients without any previous exposure to heparin [49], [50]. VITT was more common in women younger than 50 years and some of them were receiving hormonal contraceptives or estrogen-replacement therapy [49]-[51]. The pathogenesis of VITT is similar to heparin-induced thrombocytopenia; the ELISA test detected antibodies directed against the platelet factor 4 -heparin complex which activates platelets like HIT antibodies [48]-[51]. For cerebral venous sinus thrombosis, patients usually reported headache and progressive fatigue. The agreed management of this is based on the administration of IVIG or corticosteroids [52]. 


\section{LONG TERM CONSEQUENCES}

The long-term effects of COVID-19 have not been properly evaluated and determined yet. However, the likelihood of long-term subacute myocarditis following the recovery from SARS-CoV-2 infection and the development of heart failure as a long-term sequela of COVID-19 inflammatory cardiomyopathy is becoming very concerning [53]. Some clinical studies have reported the long-term consequences of COVID-19; a good deal of people is still experiencing lethargy, shortness of breath, and palpitations even after 3 to 6 months of recovery from acute infection [54], [55]. Some studies took images after a few months of recovery from COVID-19, the images demonstrated continuing manifestations of damage to the heart, even in persons who had mild COVID-19 symptoms. In another study, researchers found that after 2 months of SARS-CoV-2 detection, insistent heart involvement was noticed in $78 \%$ of survivors, among whom $60 \%$ had ongoing signs of myocarditis, which was revealed with cardiac magnetic resonance (CMR) [56]. In a CMR cohort research among 1,597 fierce athletes, clinical and subclinical myocarditis was reported in 37 athletes [57]. Evaluation with echocardiography in patients with recent COVID-19 also showed aberrations in regards of increased levels of diastolic dysfunction, decreased mean values of LV GLS, and existence of pericardial effusion, in harmony with CMR findings, as long as 2 months after recovery from COVID-19 [58], [59]. Although, the interpretation of those imaging results is at present unclear, persistent myocardial damage and fibrosis in the subacute and chronic phases after recovery advocate that COVID-19 could be an independent risk factor for heart failure development [60]. It is crucially significant to identify patients with cardiac aberrations early since cardioprotective therapy may benefit them and different follow-up methods may be needed compared to other patients.

\section{Conclusion}

From the onset of the SARS-CoV-2 outbreak with the following unparalleled health crisis, it has been a very hard and challenging time for health systems, health workers, and patients. Many clinical reports and data that have been published show that COVID-19 disease can affect the cardiovascular system. Reduced physical activity during this pandemic situation might also contribute to poor control of cardiovascular risk factors. It is clear that COVID-19 patients with existing cardiovascular diseases frequently have higher mortality and inferior prognosis. Cardiovascular diseases such as myocarditis, arrhythmias, pericardial effusion, pulmonary hypertension, and heart failure are closely related to COVID-19, and possible mechanisms of these include direct cell damage through ACE2, stress, hypoxia, inflammatory factors, and medication use. Special attention should be given to the patients with pre-existing cardiovascular conditions, and it should be made sure that adequate focus is given on protecting the cardiovascular system during the management of patients with COVID-19. A detailed management guideline should be developed both for clinicians, health workers in the hospital settings and out of hospital caregivers to protect patients with existing cardiovascular conditions and prevent COVID-19 induced cardiovascular complications before and after immunization. In that way, it will be possible to lower the fatality rates of COVID-19 patients.

\section{REFERENCES}

[1] "COVID live update: $245,121,473$ cases and 4,975,150 deaths from the Coronavirus - worldometer," Worldometers.info. [Online]. Available: https://www.worldometers.info/coronavirus/. [Accessed: 26-Oct-2021].

[2] E. A. Adeghate, N. Eid, and J. Singh, "Mechanisms of COVID-19induced heart failure: a short review," Heart Fail. Rev., vol. 26, no. 2, pp. 363-369, 2021.

[3] F. Mai, R. Del Pinto, and C. Ferri, "COVID-19 and cardiovascular diseases," J. Cardiol., vol. 76, no. 5, pp. 453-458, 2020.

[4] V. Gerc, I. Masic, N. Salihefendic, and M. Zildzic, "Cardiovascular diseases (CVDs) in COVID-19 pandemic era," Mater. Sociomed., vol. 32, no. 2, pp. 158-164, 2020.

[5] M. Boukhris et al., "Cardiovascular implications of the COVID-19 pandemic: A global perspective," Can. J. Cardiol., vol. 36, no. 7, pp. 1068-1080, 2020.

[6] D. Atri, H. K. Siddiqi, J. P. Lang, V. Nauffal, D. A. Morrow, and E. A. Bohula, "COVID-19 for the cardiologist: Basic virology, epidemiology, cardiac manifestations, and potential therapeutic strategies," JACC Basic Transl. Sci., vol. 5, no. 5, pp. 518-536, 2020.

[7] J. M. Pericàs et al., "COVID-19: from epidemiology to treatment," Eur. Heart J., vol. 41, no. 22, pp. 2092-2112, 2020.

[8] T. Guo et al., "Cardiovascular implications of fatal outcomes of patients with Coronavirus disease 2019 (COVID-19)," JAMA Cardiol., vol. 5, no. 7, pp. 811-818, 2020.

[9] O. K. Abou Hassan, C. C. Sheng, T. K. M. Wang, and P. C. Cremer, "SARS-CoV-2 myocarditis: Insights into incidence, prognosis, and therapeutic implications," Curr. Cardiol. Rep., vol. 23, no. 9, p. 129, 2021.

[10] CDC COVID-19 Response Team, "Severe outcomes among patients with Coronavirus disease 2019 (COVID-19) - United States, February 12-March 16, 2020," MMWR Morb. Mortal. Wkly. Rep., vol. 69, no. 12, pp. 343-346, 2020.

[11] T. S. M. Tsang et al., "Consecutive 1127 therapeutic echocardiographically guided pericardiocenteses: Clinical profile, practice patterns, and outcomes spanning 21 years," Mayo Clin. Proc., vol. 77, no. 5, pp. 429-436, 2002.

[12] M. Kermani-Alghoraishi, A. Pouramini, F. Kafi, and A. Khosravi, "Coronavirus disease 2019 (COVID-19) and severe pericardial effusion: From pathogenesis to management: A case report based systematic review," Curr. Probl. Cardiol., no. 100933, p. 100933, 2021.

[13] A. Rivero, A. Aceña, M. Orejas, and R. Hernandez-Estefania, "Recurrent haemorrhagic pericardial effusion due to idiopathic pericarditis: a case report," Eur. Heart J. Case Rep., vol. 3, no. 1, p. ytz018, 2019.

[14] F. Sauer, C. Dagrenat, P. Couppie, G. Jochum, and P. Leddet, "Pericardial effusion in patients with COVID-19: case series," Eur. Heart J. Case Rep., vol. 4, no. FI1, pp. 1-7, 2020.

[15] K. E. Parsova, L. Pay, Y. Oflu, R. Hacıyev, and G. Çinier, "A rare presentation of a patient with COVID-19: Cardiac tamponade," Turk Kardiyol. Dern. Ars., vol. 48, no. 7, pp. 703-706, 2020.

[16] M. F. Dabbagh, L. Aurora, P. D’Souza, A. J. Weinmann, P. Bhargava, and M. B. Basir, "Cardiac tamponade secondary to COVID-19," JACC Case Rep, vol. 2, no. 9, pp. 1326-1330, 2020.

[17] C. Ejikeme, M. Gonzalez, S. Elkattawy, R. Alyacoub, and C. Sherer, "Subacute COVID-19 infection presenting as indolent large pericardial effusion," Cureus, vol. 12, no. 10, p. e10769, 2020.

[18] A. Reddy, S. Nethercott, R. Duehmke, S. Nair, and O. Abdul-Samad, "“dry' pericarditis with rapid progression to tamponade as a feature of COVID-19," European Medical Journal, 2021.

[19] M. Imazio et al., "Anti-inflammatory therapies for pericardial diseases in the COVID-19 pandemic: safety and potentiality: Safety and potentiality," J. Cardiovasc. Med. (Hagerstown), vol. 21, no. 9, pp. 625-629, 2020.

[20] F. Kafi, A. Bolourian, Z. Mojtahedi, and A. Pouramini, "High mobility group box 1 (HMGB1) in COVID-19," J. Prev. Epidemiol., vol. 6, no. 1, pp. e11-e11, 2021. 
[21] H. Karmouty-Quintana, R. A. Thandavarayan, S. P. Keller, S. Sahay, L. M. Pandit, and B. Akkanti, "Emerging mechanisms of pulmonary vasoconstriction in SARS-CoV-2-induced acute respiratory distress syndrome (ARDS) and potential therapeutic targets," Int. J. Mol. Sci., vol. 21 , no. 21 , p. $8081,2020$.

[22] S. Kache et al., "COVID-19 PICU guidelines: for high- and limitedresource settings," Pediatr. Res., vol. 88, no. 5, pp. 705-716, 2020.

[23] "ESC guidance for the diagnosis and management of CV disease during the COVID-19 pandemic," Escardio.org. [Online]. Available: https://www.escardio.org/Education/COVID-19-andCardiology/ESC-COVID-19-Guidance. [Accessed: 26-Oct-2021].

[24] H. M. Al-Kuraishy, A. I. Al-Gareeb, M. S. Al-Niemi, A. K. AlBuhadily, N. A. Al-Harchan, and C. Lugnier, "COVID-19 and phosphodiesterase enzyme type 5 inhibitors," J. Microsc. Ultrastruct., vol. 8, no. 4, pp. 141-145, 2020.

[25] W.-X. Feng, Y. Yang, J. Wen, Y.-X. Liu, L. Liu, and C. Feng, "Implication of inhaled nitric oxide for the treatment of critically ill COVID-19 patients with pulmonary hypertension," ESC Heart Fail., vol. 8, no. 1, pp. 714-718, 2021.

[26] B. Yu, M. J. Raher, G. P. Volpato, K. D. Bloch, F. Ichinose, and W. M. Zapol, "Inhaled nitric oxide enables artificial blood transfusion without hypertension," Circulation, vol. 117, no. 15, pp. 1982-1990, 2008.

[27] M. P. Nägele, B. Haubner, F. C. Tanner, F. Ruschitzka, and A. J. Flammer, "Endothelial dysfunction in COVID-19: Current findings and therapeutic implications," Atherosclerosis, vol. 314, pp. 58-62, 2020.

[28] D. Wang et al., "Clinical characteristics of 138 hospitalized patients with 2019 novel Coronavirus-infected pneumonia in Wuhan, China," JAMA, vol. 323, no. 11, pp. 1061-1069, 2020.

[29] A. R. Akkawi and M. Ghazal, "COVID-19 and cardiac arrhythmias: A review of the literature," Cureus, 2021.

[30] T.-Y. Xiong, S. Redwood, B. Prendergast, and M. Chen, "Coronaviruses and the cardiovascular system: acute and long-term implications," Eur. Heart J., vol. 41, no. 19, pp. 1798-1800, 2020.

[31] M. Traebert, B. Dumotier, L. Meister, P. Hoffmann, M. DominguezEstevez, and W. Suter, "Inhibition of hERG K+ currents by antimalarial drugs in stably transfected HEK293 cells," Eur. J. Pharmacol., vol. 484, no. 1, pp. 41-48, 2004.

[32] J. Demazière, J. M. Fourcade, C. T. Busseuil, P. Adeleine, S. M. Meyer, and J. M. Saïssy, "The hazards of chloroquine self prescription in west Africa," J. Toxicol. Clin. Toxicol., vol. 33, no. 4, pp. 369-370, 1995.

[33] A. Cervera, G. Espinosa, J. Font, and M. Ingelmo, "Cardiac toxicity secondary to long term treatment with chloroquine," Ann. Rheum. Dis., vol. 60 , no. 3 , p. $301,2001$.

[34] J. Carron, Z. Sharif, H. Hussein, M. Kennedy, B. McAdam, and R. Sheahan, "Clinical guidance for navigating the QTc-prolonging and arrhythmogenic potential of pharmacotherapy during the COVID-19 pandemic," Ir. J. Med. Sci., vol. 190, no. 1, pp. 403-409, 2021.

[35] H. Onohuean, H. M. Al-Kuraishy, A. I. Al-Gareeb, S. Qusti, E. M. Alshammari, and G. E.-S. Batiha, "Covid-19 and development of heart failure: mystery and truth," Naunyn. Schmiedebergs. Arch. Pharmacol., vol. 394, no. 10, pp. 2013-2021, 2021.

[36] F. Zhou et al., "Clinical course and risk factors for mortality of adult inpatients with COVID-19 in Wuhan, China: a retrospective cohort study," Lancet, vol. 395, no. 10229, pp. 1054-1062, 2020.

[37] X. Peng et al., "Promising therapy for heart failure in patients with severe COVID-19: Calming the cytokine storm," Cardiovasc. Drugs Ther., vol. 35, no. 2, pp. 231-247, 2021.

[38] C. Burkard et al., "ATP1A1-mediated Src signaling inhibits coronavirus entry into host cells," J. Virol., vol. 89, no. 8, pp. 44344448, 2015.

[39] K. Ihenetu, R. Espinosa, R. de Leon, G. Planas, A. Perez-Pinero, and L. Waldbeser, "Digoxin and digoxin-like immunoreactive factors (DLIF) modulate the release of pro-inflammatory cytokines," Inflamm. Res., vol. 57, no. 11, pp. 519-523, 2008.

[40] J. Lee et al., "Digoxin ameliorates autoimmune arthritis via suppression of Th17 differentiation," Int. Immunopharmacol., vol. 26, no. 1 , pp. 103-111, 2015.

[41] C. Skayem and N. Ayoub, "Carvedilol and COVID-19: A potential role in reducing infectivity and infection severity of SARS-CoV2," Am. J. Med. Sci., vol. 360, no. 3, p. 300, 2020.

[42] T. Kurum, E. Tatli, and M. Yuksel, "Effects of carvedilol on plasma levels of pro-inflammatory cytokines in patients with ischemic and nonischemic dilated cardiomyopathy," Tex. Heart Inst. J., vol. 34, no. 1, pp. 52-59, 2007.

[43] K. Kotfis et al., "COVID-19-the potential beneficial therapeutic effects of spironolactone during SARS-CoV-2 infection," Pharmaceuticals (Basel), vol. 14, no. 1, p. 71, 2021.

[44] B. Bozkurt, I. Kamat, and P. J. Hotez, "Myocarditis with COVID-19 mRNA vaccines," Circulation, vol. 144, no. 6, pp. 471-484, 2021.

[45] Coronaviridae Study Group of the International Committee on Taxonomy of Viruses, "The species Severe acute respiratory syndrome-related coronavirus: classifying 2019-nCoV and naming it SARS-CoV-2," Nat. Microbiol., vol. 5, no. 4, pp. 536-544, 2020.

[46] M. Marshall et al., "Symptomatic acute myocarditis in 7 adolescents after Pfizer-BioNTech COVID-19 vaccination," Pediatrics, vol. 148, no. 3, p. e2021052478, 2021.

[47] B. J. Maron et al., "Eligibility and disqualification recommendations for competitive athletes with cardiovascular abnormalities: Task force 3: Hypertrophic cardiomyopathy, arrhythmogenic right ventricular cardiomyopathy and other cardiomyopathies, and myocarditis: A scientific statement from the American heart association and American college of cardiology," J. Am. Coll. Cardiol., vol. 66, no. 21, pp. 2362 2371, 2015.

[48] D. B. Cines and J. B. Bussel, "SARS-CoV-2 vaccine-induced immune thrombotic thrombocytopenia," N. Engl. J. Med., vol. 384, no. 23, pp. 2254-2256, 2021.

[49] A. Greinacher, T. Thiele, T. E. Warkentin, K. Weisser, P. A. Kyrle, and S. Eichinger, "Thrombotic thrombocytopenia after ChAdOx1 nCov-19 vaccination," N. Engl. J. Med., vol. 384, no. 22, pp. 20922101, 2021.

[50] K.-L. Muir, A. Kallam, S. A. Koepsell, and K. Gundabolu, "Thrombotic thrombocytopenia after Ad26.COV2.S vaccination," $N$. Engl. J. Med., vol. 384, no. 20, pp. 1964-1965, 2021.

[51] I. See et al., "US case reports of cerebral venous sinus thrombosis with thrombocytopenia after Ad26.COV2.S vaccination, March 2 to April 21, 2021,"JAMA, vol. 325, no. 24, pp. 2448-2456, 2021.

[52] K. L. Furie, M. Cushman, M. S. V. Elkind, P. D. Lyden, G. Saposnik, and American Heart Association/American Stroke Association Stroke Council Leadership, "Diagnosis and management of cerebral venous sinus thrombosis with vaccine-induced immune thrombotic thrombocytopenia," Stroke, vol. 52, no. 7, pp. 2478-2482, 2021.

[53] L. Italia et al., "COVID-19 and heart failure: From epidemiology during the pandemic to myocardial injury, myocarditis, and heart failure sequelae," Front. Cardiovasc. Med., vol. 8, p. 713560, 2021.

[54] C. Huang et al., "6-month consequences of COVID-19 in patients discharged from hospital: a cohort study," Lancet, vol. 397, no. 10270, pp. 220-232, 2021.

[55] B. van den Borst et al., "Comprehensive health assessment 3 months after recovery from acute Coronavirus disease 2019 (COVID19)," Clin. Infect. Dis., vol. 73, no. 5, pp. e1089-e1098, 2021.

[56] V. O. Puntmann et al., "Outcomes of cardiovascular magnetic resonance imaging in patients recently recovered from Coronavirus disease 2019 (COVID-19)," JAMA Cardiol., vol. 5, no. 11, pp. 12651273, 2020.

[57] C. J. Daniels et al., "Prevalence of clinical and subclinical myocarditis in competitive athletes with recent SARS-CoV-2 infection: Results from the big Ten COVID-19 Cardiac Registry: Results from the big ten COVID-19 cardiac registry," JAMA Cardiol., vol. 6, no. 9, pp. 1078-1087, 2021.

[58] D. Brito et al., "High prevalence of pericardial involvement in college student athletes recovering from COVID-19," JACC Cardiovasc. Imaging, vol. 14, no. 3, pp. 541-555, 2021.

[59] L. T. Weckbach et al., "Myocardial inflammation and dysfunction in COVID-19-associated myocardial injury," Circ. Cardiovasc. Imaging, vol. 14, no. 1, p. e012220, 2021.

[60] G. Zaccone, D. Tomasoni, L. Italia, C. M. Lombardi, and M. Metra, "Myocardial involvement in COVID-19: An interaction between comorbidities and heart failure with preserved ejection fraction. A further indication of the role of inflammation," Curr. Heart Fail. Rep., vol. 18, no. 3, pp. 99-106, 2021. 